Pacific Journal of Mathematic 


\title{
QUOTIENTS OF COMPLETE MULTIPARTITE GRAPHS
}

\author{
CHO WeI SiT
}

\begin{abstract}
The principal result of this paper is the determination of every graph that can be covered by a complete multipartite graph, in the usual topological sense of covering spaces.
\end{abstract}

Various papers of S.R. Alpert, J.L. Gross, and T.W. Tucker have explicitly recognized that knowing what a given graph covers is helpful in constructing surface imbeddings for it, acknowledging that this approach is the underlying secret in the combinatorial current graph method of W. Gustin. Moreover, the fact that every Cayley graph covers a bouquet of circles is of implicit importance in other work on Cayley graph imbeddings, such as that of A.T. White on the genus of a group.

1. Introduction. The present paper is a sequel to the work of Gross and Tucker [2], whose terminology is adopted here. In addition, the following notations are used.

Let $G$ be a graph. Then $V(G)$ and $E(G)$ denote respectively the set of vertices and the set of edges of $G$.

A k-partite graph is a graph whose vertices can be partitioned into $k$ cells such that no two vertices within the same cell have an edge adjoining them. In general, it is tedious to determine for a given graph $G$ the minimum number $k$ such that $G$ is $k$-partite, which is, of course, the chromatic number of $G$. The complete $k$-partite graph $K_{n_{1}, \cdots, n_{k}}$ is the (k-partite) graph with a vertex set partition $V_{1}, \cdots, V_{k}$ such that $\left|V_{i}\right|=n_{i}$ for $i=1, \cdots, k$, and for each $u \in V_{i}, v \in V_{j}$, there is an edge between $u$ and $v$ if and only if $i \neq j$.

Given two graphs $G_{1}$ and $G_{2}$, the notation $G_{1}(+, d) G_{2}$ means the $d$-fold suspension of $G_{1}$ and $G_{2}$, which is defined to be the smallest graph which contains both $G_{1}$ and $G_{2}$ and such that for every pair of vertices $(u, v)$ with $u \in V\left(G_{1}\right), v \in V\left(G_{2}\right)$, there are exactly $d$-edges between $u$ and $v$. The 1 -fold suspension of $G_{1}$ and $G_{2}$ is also denoted by $G_{1}+G_{2}$, and is elsewhere called the "join" of $G_{1}$ and $G_{2}$.

A graph map $p: K \rightarrow K^{\prime}$ is called a $d$-fold pseudocovering if the inverse image of each point and of each (open) line of $K^{\prime}$ has $d$ components in $K$ and if for every vertex $v$ of $K$ the degree of $v$ equals the degree of its image $p(v)$. In such a case, the graph $K^{\prime}$ is said to be a $d$-fold pseudoquotient of $K$.

A graph map $p: K \rightarrow K^{\prime}$ such that $K^{\prime}$ is connected is called a 
covering if every point $v$ of $K$ has a topological neighborhood which is mapped one-one onto its image in $K^{\prime}$. (Since $K^{\prime}$ is compact, this agrees with the usual definition of a covering.) In such a case, the graph $K^{\prime}$ is said to be a $d$-fold quotient of $K$.

It can be shown that every covering is also a pseudocovering (e.g. using Lemma 3.4 of W.S. Massey [3]), and therefore that every quotient is a pseudoquotient. What makes the determination of quotients of complete multipartite graphs somewhat harder than that of complete graphs is that whereas every pseudoquotient of a complete graph is a quotient, no analogous result holds for complete multipartite graphs. The following example illustrates this difference.

Example. Consider the symmetric bipartite graph $K_{6,6}$.

$$
\text { Let } V_{1}=\left\{u_{1}, u_{2}, v_{1}, v_{2}, v_{3}, v_{4}\right\} \text {, }
$$

and

$$
V_{2}=\left\{u_{3}, u_{4}, w_{1}, w_{2}, w_{3}, w_{4}\right\}
$$

be the cells of the associated partition of vertices of $K_{6,6}$.

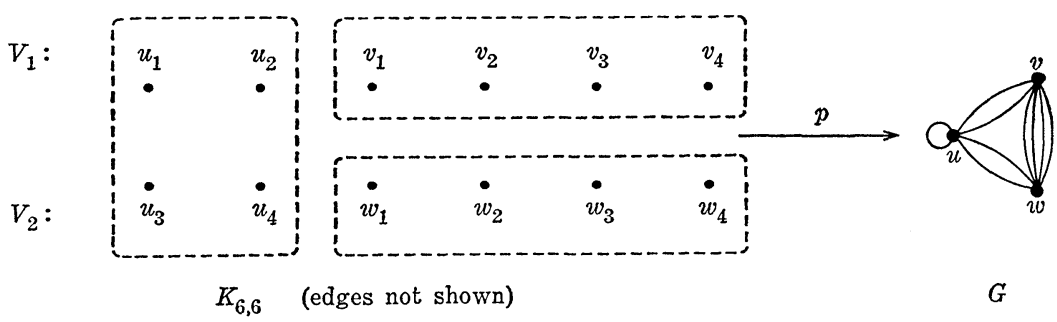

Figure 1. A pseudoquotient of $K_{6,6}$ that is not a quotient.

Define a graph map $p$ from $K_{6,6}$ to the graph $G$ in Figure 1 by "dropping the subscript" and such that four edges are mapped to one. The graph map $p$ is obviously a 4-fold pseudocovering, and therefore $G$ is a pseudoquotient of $K_{6,6}$. The following argument, however, shows that $G$ is not a quotient of $K_{6,6}$.

Suppose to the contrary that there exists a graph map $f: K_{6,6} \rightarrow G$ that is a 4 -fold covering. Then, since the vertex $v$ has no selfadjacency, the fibre over $v$ (i.e. $f^{-1}(v)$ ) must lie entirely in one cell of the partition, say $V_{1}$. For similar reasons, the fibre over the vertex $w$ is contained in the partition cell $V_{2}$. Hence, after some reindexing if necessary, the map $f$ is identical to $p$ on the set of vertices. However, the four edges adjoining the vertex $u_{4}$ to the vertices $v_{1}, v_{2}, v_{3}$, and $v_{4}$ have to be mapped into the two edges 
adjoining $u$ and $v$, establish ing that the map cannot be a local homeomorphism, and consequently not a covering. This proves that the graph $G$ is not a 4 -fold quotient of $K_{6,6}$.

A generalization of the above counting argument utilizing the local homeomorphism property is used to distinguish the quotients from the pseudoquotients of the complete $k$-partite graph $K_{n_{1}, \ldots, n_{k}}$. That generalization and the constructive method of reduced voltage graphs are sufficient for a complete determination of all quotients of $K_{n_{1}, \ldots, n_{k}}$.

2. On the homogeneity of fibres. The local homeomorphism property is now applied to prove two lemmas which are the keys to distinguishing between quotients and mere pseudoquotients of $K_{n_{1}, \ldots, n_{k}}$.

LEMmA 1. Let $V_{1}, \cdots, V_{k}$ be the associated vertex partition cells of the complete k-partite graph $K_{n_{1}, \ldots, n_{k}}$. Let the graph map p: $K_{n_{1}, \ldots, n_{k}} \rightarrow G$ be a d-fold covering. Let $v$ be a vertex of $G$ and let $V_{i_{1}}, \cdots, V_{i_{t}}$ be the cells which have non-empty intersection with the fibre $p^{-1}(v)$. Then the number $\left|p^{-1}(v) \cap V_{i_{r}}\right|$ is the same for all cells $V_{i_{r}}, r=1, \cdots, t$.

Proof. For $r=1, \cdots, t$, define $m_{r}$ to be the number $\mid p^{-1}(v) \cap$ $V_{i_{r}} \mid$ of points in which the fibre over the vertex $v$ meets the partition cell $V_{i_{r}}$. Since $d=\left|p^{-1}(v)\right|$, it follows that $d=\sum_{r=1}^{t} m_{r}$. Since $K_{n_{1}, \ldots, n_{k}}$ is complete $k$-partite, there are $\sum_{i<j} m_{i} m_{j}$ edges between vertices in the fibre $p^{-1}(v)$, from which it follows that there are precisely $\left(\sum_{i<j} m_{i} m_{j}\right) / d$ loops at the vertex $v$. On the other hand, for any $r=1, \cdots, t$, if $u$ is a vertex in $p^{-1}(v) \cap V_{i_{r}}$, then there are $\sum_{i \neq r} m_{i}$ edges adjoining $u$ to other vertices of $p^{-1}(v)$. Since the covering $p$ is a local homeomorphism, it follows that there are $\sum_{i \neq r} m_{i}$ loops at $v$. Thus, $\left(\sum_{i<j} m_{i} m_{j}\right) / d=\sum_{i \neq r} m_{i}=d-m_{r}$. The left-hand formula is independent of $r$, so the right side must also be independent of $r$. Hence, the numbers $m_{r}$ do not differ for different cells $V_{i_{r}}$, proving the lemma.

Let $V_{1}, \cdots, V_{k}$ be the associated vertex partition cells of the complete $k$-partite graph $K_{n_{1}, \ldots, n_{k}}$. Let $p: K_{n_{1}, \ldots, n_{k}} \rightarrow G$ be a covering, and $v \in V(G)$. The vertex $v$ is said to have fibre type $\left\{i_{1}, \cdots, i_{t}\right\}$ if $V_{i_{1}}, \cdots, V_{i_{t}}$ are the cells of partition which have nonempty intersection with the fibre $p^{-1}(v)$. In such a case, the fibre $p^{-1}(v)$ is said to be of type $\left\{i_{1}, \cdots, i_{t}\right\}$. A fibre is said to be homogeneous if it is contained in one cell of partition, that is, if its type contains only one element. 
Lemma 2. Let $V_{1}, \cdots, V_{k}$ be the vertex partition cells of the complete k-partite graph $K_{n_{1}, \ldots, n_{k}}$. Let $p: K_{n_{1}, \ldots, n_{k}} \rightarrow G$ be a $d$-fold covering. Then the fibre types of any two vertices of $G$ are either disjoint or identical.

Proof. Suppose that vertices $u$ and $v$ of the graph $G$ have nondisjoint fibre types. Suppose also that vertex $v$ has fibre type $\left\{i_{1}, \cdots, i_{t}\right\}$ and that $i_{1}$ is also contained in the fibre type of $u$. To obtain a contradiction, suppose further that $p^{-1}(u) \cap V_{i_{j}}=\varnothing$ for some cell $V_{i_{j}}$ such that $t \geqq j>1$, and that $\widetilde{v}$ is a vertex in $p^{-1}(v) \cap V_{i_{j}}$. There is an edge between $\widetilde{v}$ and every vertex in $p^{-1}(u)$, so there is a total of $d$ edges between $\widetilde{v}$ and vertices in $p^{-1}(u)$. Since both the fibres $p^{-1}(u)$ and $p^{-1}(v)$ have nonempty intersection with $V_{i_{1}}$, the total number of edges adjoining vertices in $p^{-1}(u)$ to vertices in $p^{-1}(v)$ is less than $d^{2}$, and therefore the number of edges between $u$ and $v$ is less than $d$, contradicting the fact that the covering $p$ is a local homeomorphism at the vertex $\widetilde{v}$. Hence, the fibre $p^{-1}(u)$ must have nonempty intersection with the cell $V_{i_{j}}$, for all $j=$ $1, \cdots, t$, that is, the fibre type of $v$ is contained in the fibre type of $u$. Reversing the roles of $u$ and $v$ completes the proof of the lemma.

REMARK 1. It follows from Lemma 1 and Lemma 2 that if the covering $p: K_{n_{1}, \ldots, n_{k}} \rightarrow G$ has some fibre of type $\left\{i_{1}, \cdots, i_{t}\right\}$, then $n_{i_{1}}=n_{i_{2}}=\cdots=n_{i_{t}}$.

Given a graph $G$, the $d$-fold $G$, denoted by ${ }^{d} G$, is the smallest graph having the same vertex set as $G$, and such that for every edge between a pair of vertices $u$ and $v$ in $G$, there are exactly $d$ edges adjoining $u$ and $v$ in ${ }^{d} G$.

The reduced voltage graph construction of Gross and Tucker [2] is now applied to classify all the quotients of the complete $k$-partite graph that have only homogeneous fibres.

THEOREM 1. For each common divisor $d$ of $\left\{n_{1}, \cdots, n_{k}\right\}$, the $d$ fold complete $k$-partite graph ${ }^{d} K_{n_{1} / d, \ldots, n_{k} / d}$ is a $d$-fold quotient of $K_{n_{1}, \ldots, n_{k}}$ with all fibres homogeneous. Furthermore, the graph $K_{n_{1}, \ldots, n_{k}}$ has no other quotients with only homogeneous fibres.

Proof (Existence). Let $u$ and $v$ be vertices belonging to different cells in the graph ${ }^{d} K_{n_{1} / d, \ldots, n_{k} / d}$. The $d$ edges running from $u$ to $v$ are bijectively assigned the voltages $0,1, \cdots, d$-1 modulo $d$ (assuring that their respective reverses are assigned the voltages $0, d-1, \cdots, 1$ 
modulo d). The corresponding derived graph has no multiple or self adjacencies, and its vertices are partitioned into $k$ cells according to their images under the covering projection, making it $k$-partite and assuring homogeneity of all fibres. By counting edges, one shows that the derived graph is actually complete $k$-partite.

(Uniqueness) If the graph map $p: K_{n_{1},,_{k}} \rightarrow G$ is a $d$-fold covering with only homogeneous fibres, then the vertices of $G$ can evidently be partitioned into $k$-cells according to their inverse images under the map $p$, making $G$ a $k$-partite graph. If the vertices $u$ and $v$ of $G$ lie in different cells of the associated partition, then there are $d^{2}$ edges between the $d$ vertices of $P^{-1}(u)$ and the $d$ vertices of $P^{-1}(v)$, and consequently $d$ edges between $u$ and $v$, proving that the graph $G$ is the $d$-fold complete $k$-partite graph ${ }^{d} K_{n_{1} / d, \ldots, n_{k} / d}$.

3. Quotients of symmetric multipartite graphs. The following lemma gives the necessary and sufficient conditions for the existence and uniqueness of a special type of quotient of the symmetric $k$ partite graph $K_{n, \ldots, n}$.

Lemma 3. Let the graph $G$ be a d-fold quotient of the symmetric $k$-partite graph $K_{n,}$, , which has at least one fibre of type $\{1,2, \cdots, k\}$. Then all fibres are of type $\{1,2, \cdots, k\}$.

Furthermore, the following conditions are satisfied:

(i) $k$ divides $d$.

(ii) If $c=d / k$, then $c$ divides $n$ and $c(k-1) / 2$ is an integer.

Moreover, the graph $G$ is realized by amalgamating a bouquet of $c(k-1) / 2$ circles to every vertex of the graph ${ }^{c(k-1)} K_{n / c}$.

Conversely, given integers $d$ and $k$ satisfying (i) and (ii), there exists a $d$-fold quotient of the symmetric $k$-partite graph $K_{n, \ldots, n}$ with all fibres of type $\{1,2, \cdots, k\}$.

Proof. Since no fibre type can be disjoint from the type $\{1,2, \cdots, k\}$, Lemma 2 implies that all fibres are type $\{1, \cdots, k\}$. According to Lemma 1 , the $d$ vertices of any fibre are distributed in equal number among the partition cells $V_{1}, \cdots, V_{k}$, so $k$ divides $d$, which is precisely property (i). There are $c$ vertices in the intersection of a fibre with a cell and there are $n$ vertices in each cell. Hence, $c$ divides $n$. Since there are $c^{2} k(k-1) / 2$ edges between vertices within each fibre, there are $c(k-1) / 2$ loops at each vertex of $G$, proving that $c(k-1) / 2$ is an integer. Let $u$ and $v$ be different vertices of $G$. For each vertex in the fibre over $u$, there are $c(k-1)$ edges adjoining it to a vertex in the fibre over $v$, hence 
there are $c(k-1)$ edges adjoining $u$ and $v$, proving that $G$ is as described in the lemma.

To prove the converse, let $L$ be a graph obtained by amalgamating a bouquet of $c(k-1) / 2$ circles to the graph ${ }^{c(k-1)} K_{n / c}$. At each vertex of $L$, give each of the circles a preferred direction and assign the voltages $1,2, \cdots, k-1, \hat{k}, k+1, \cdots, 2 \hat{k}, \cdots, 3 \hat{k}, \cdots$, modulo $d$ until the circles at that vertex are exhausted, where the notation $\hat{j}$ denotes that $j$ is deleted from the sequence. Since there are only $c(k-1) / 2$ circles at each vertex, only voltages up to [ck/2] are assigned, (where the notation $[x]$ denotes the greatest integer less than or equal to $x$ ), guaranteeing that no two voltages are inverses to each other which is necessary to ensure that the derived graph has no multiple adjacencies. The $c(k-1)$ edges in the graph $L$ running from any vertex $v$ to any other vertex $u$ are assigned the voltages $1,2, \cdots, k-1, \hat{k}, k+1, \cdots, 2 \hat{k}, \cdots, c k-1$ modulo $d$ (once again assuring that their respective reverses are assigned the respective inverses as voltages). The vertices of the derived graph are then partitioned into $k$ cells according to the equivalence classes of their second components modulo $k$. Since voltages congruent to zero modulo $k$ are not present, the derived graph is $k$-partite. By a counting argument similar to that in the proof of Theorem 1 , it is proved that the derived graph is actually complete $k$-partite.

The following lemma which gives a quotient for the suspension of two derived graphs with voltages in the same group is proved using arguments sufficiently similar to those for Lemma 3, and the proof is omitted.

LEMmA 4. Let $\left(G_{1}, \beta_{1}\right)$ and $\left(G_{2}, \beta_{2}\right)$ be reduced voltage graphs with voltages in a group $G$ of order $d$. Let the set $\left\{x_{1}, \cdots, x_{d}\right\}$ be the set of elements of the group $G$. Let the reduced voltage graph $\left(G_{1}(+, d) G_{2}, \beta\right)$ be defined by:

$$
\beta(e)= \begin{cases}\beta_{i}(e) & \text { if } e \text { is an oriented edge in } G_{i}, i=1,2 . \\ x_{j} & \text { if } e \text { is the } j^{\text {th }} \text { oriented edge adjoining a } \\ & \text { vertex in } G_{1} \text { to a vertex in } G_{2} .\end{cases}
$$

Then their derived graphs satisfy the following condition:

$$
\left(G_{1}(+, d) G_{2}\right)^{\beta} \cong G_{1}^{\beta_{1}}+G_{2}^{\beta_{2}} \text {. }
$$

Conversely, let $G_{1}^{\prime}$ and $G_{2}^{\prime}$ be arbitrary graphs. Then any $d$ fold quotient of the graph $G_{1}^{\prime}+G_{2}^{\prime}$ is isomorphic to the $d$-fold suspension $G_{1}(+, d) G_{2}$ where the graphs $G_{1}$ and $G_{2}$ are some $d$-fold quotients of the graphs $G_{1}^{\prime}$ and $G_{2}^{\prime}$ respectively. 
4. Characterization of quotients of complete $k$-partite graphs. Given any $d$-fold covering $p: K_{n_{1}, \ldots, n_{k}} \rightarrow G$, according to Lemma 2 the collection of all the various fibre types partitions the set $\{1, \cdots, k\}$. Reindex if necessary, assume them to be

$$
\begin{aligned}
& \left\{1,2, \cdots, k_{1}\right\}, \quad \text { (type 1) } \\
& \left\{k_{1}+1, k_{1}+2, \cdots, k_{1}+k_{2}\right\}, \quad(\text { type 2) } \\
& \cdot \cdot \cdot \cdot \cdot \cdot \cdot \cdot \\
& \cdot \cdot \cdot \cdot \cdot \cdot \cdot \cdot \\
& \left\{k_{1}+\cdots+k_{j-1}+1, \cdots, k_{1}+\cdots+k_{j-1}+k_{j}\right\}, \quad \text { (type } j \text { ) } \\
& \left\{k_{1}+\cdots+k_{j}+1\right\}, \\
& \left\{k_{1}+\cdots+k_{j}+2\right\}, \\
& \cdot \cdot \cdot \cdot \cdot \cdot \cdot \cdot \cdot \\
& \cdot \cdot \cdot \cdot \cdot \cdot \cdot \cdot . \\
& \{k\} .
\end{aligned}
$$

From Remark 1, it follows that

$$
\begin{aligned}
& n_{1}=n_{2}=\cdots=n_{k_{1}}, \\
& n_{k_{1}+1}=n_{k_{1}+2}=\cdots=n_{k_{1}+k_{2}} \\
& \cdot \cdot \cdot \cdot \cdot \cdot \cdot \cdot \cdot \cdot \\
& \cdot \cdot \cdot \cdot \cdot \cdot \cdot \cdot \cdot \\
& \text { etc. }
\end{aligned}
$$

Therefore the complete $k$-partite graph $K_{n_{1}, \ldots, n_{k}}$ can be decomposed as follows:

$$
\begin{aligned}
& K_{n_{1}, \ldots, n_{k t}} \\
& =K_{m_{1}, \ldots, m_{1}}+K_{k_{1} \text { indiees }} K_{m_{2}, \ldots, m_{2}}+\cdots+K_{k_{2} \text { indices }} \underbrace{}_{m_{j}, \ldots, m_{j}}+K_{m_{j+1}, \ldots, m_{t}},
\end{aligned}
$$

where

$$
m_{1}=n_{1}, m_{2}=n_{k_{1}+1}, m_{3}=n_{k_{1}+k_{2}+1}, \cdots, m_{t}=n_{k}
$$

THEOREM 2. Let the graph $G$ be a d-fold quotient of the complete $k$-partite graph $K_{n_{1}, \ldots, n_{k}}$ with fibre types as described above. Then the graph $K_{n_{1}, \ldots, n_{k}}$ can be decomposed as in (*). Furthermore, the following are satisfied:

(i) $d$ divides $\sum_{i=1}^{k} n_{i}$.

(ii) For all $i=1, \cdots, j$, the cardinality $k_{i}$ of fibre type $i$ divides $d$, and if $d=c_{i} k_{i}$, then $c_{i}$ divides $m_{i}$ and $c_{i}\left(k_{i}-1\right) / 2$ is an integer.

(iii) $d$ divides $m_{h}$ for every $h$ such that $j<h \leqq t$. Moreover, the 
graph $G$ is isomorphic to the graph $G_{1}(+, d) G_{2}(+, d) \cdots(+, d) G_{j}(+, d) G^{*}$, where $G_{i}$ is the graph $c_{i}\left(k_{i}-1\right) K_{m_{i} / c_{i}}$ with $c_{i}\left(k_{i}-1\right) / 2$ circles amalgamated at each vertex and $G^{*}$ is the graph ${ }^{d} K_{m_{j+1} / d, \ldots, m_{t} / d}$.

Conversely, given a complete $k$-partite graph $K_{n_{1}, \ldots, n_{k}}$ that can be decomposed as in $\left({ }^{*}\right)$, and an integer $d$ satisfying (i), (ii), (iii), then a $d$-fold quotient of $K_{n_{1}, \ldots, n_{k}}$ with fibre types as described exists.

Proof. Condition (i) follows from the property of $d$-fold quotients. All the rest follows from repeated applications of Lemma 4 utilizing what is known from Theorem 1 and Lemma 3.

REMARK 2. Since the complete graph $K_{n}$ can be considered as the symmetric $n$-partite graph $K_{1, \ldots, 1}$, Therem 2 extends the result in [2].

REMARK 3. For the complete bipartite graph $K_{n_{1}, n_{2}}$, quotients with nonhomogeneous fibres exist only when $n_{1}=n_{2}$.

Acknowledgment. The author wishes to thank Professor J.L. Gross for suggesting the problem and many valuable comments.

\section{REFERENCES}

1. J. L. Gross and S. R. Alpert, The topological theory of current graphs, J. Combinatorial Theory (B) 17 (1974), 218-233.

2. J. L. Gross and T. W. Tucker, Quotients of complete graphs: Revisiting the Heawood map-coloring problem, Pacific J. Math. 55 (1974), 391-402.

3. W. S. Massey, Algebraic Topology: An Introduction, Harcourt, Brace and World, New York, 1967.

4. A. T. White, On the genus of a group, Trans. Amer. Math. Soc. 173 (1972), 203-214.

Received October 3,1975 . The preparation of this manuscript was partially supported by the National Science Foundation under contract MPS74-05481 A01 at Columbia University.

Columbia University 


\title{
PACIFIC JOURNAL OF MATHEMATICS
}

\author{
EDITORS
}

\author{
RICHARD ARENS (Managing Editor) \\ University of California \\ Los Angeles, California 90024
}

\author{
R. A. Beaumont \\ University of Washington \\ Seattle, Washington 98105
}

J. DugundjI

Department of Mathematics

University of Southern California

Los Angeles, California 90007

D. Gilbarg and J. Milgram

Stanford University

Stanford, California 94305

\section{ASSOCIATE EDITORS}
E. F. BECKENBACH
B. H. NEUMANN
F. WOLF
K. Yoshida

\section{SUPPORTING INSTITUTIONS}

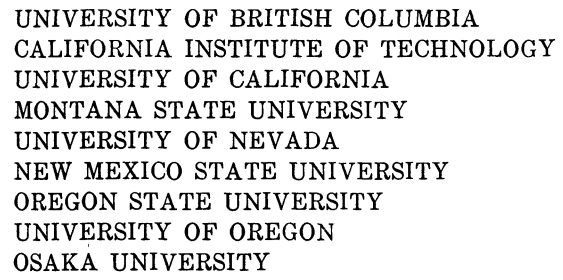

UNIVERSITY OF BRITISH COLUMBIA CALIFORNIA INSTITUTE OF TECHNOLOGY UNIVERSITY OF CALIFORNIA MONTANA STATE UNIVERSITY UNIVERSITY OF NEVADA NEW MEXICO STATE UNIVERSITY OREGON STATE UNIVERSITY UNIVERSITY OF OREGON OSAKA UNIVERSITY

\author{
UNIVERSITY OF SOUTHERN CALIFORNIA \\ STANFORD UNIVERSITY \\ UNIVERSITY OF HAWAII \\ UNIVERSITY OF TOKYO \\ UNIVERSITY OF UTAH \\ WASHINGTON STATE UNIVERSITY \\ UNIVERSITY OF WASHINGTON

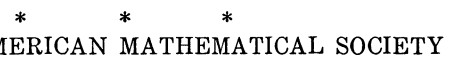

The Supporting Institutions listed above contribute to the cost of publication of this Journal, but they are not owners or publishers and have no responsibility for its content or policies.

Mathematical papers intended for publication in the Pacific Journal of Mathematics should be in typed form or offset-reproduced, (not dittoed), double spaced with large margins. Please do not use built up fractions in the text of your manuscript. You may however, use them in the displayed equations. Underline Greek letters in red, German in green, and script in blue. The first paragraph or two must be capable of being used separately as a synopsis of the entire paper. Items of the bibliography should not be cited there unless absolutely necessary, in which case they must be identified by author and Journal, rather than by item number. Manuscripts, in triplicate, may be sent to any one of the editors. Please classify according to the scheme of Math. Reviews, Index to Vol. 39. All other communications should be addressed to the managing editor, or Elaine Barth, University of California, Los Angeles, California, 90024.

The Pacific Journal of Mathematics expects the author's institution to pay page charges, and reserves the right to delay publication for nonpayment of charges in case of financial emergency.

100 reprints are provided free for each article, only if page charges have been substantially paid. Additional copies may be obtained at cost in multiples of 50 .

The Pacific Journal of Mathematics is issued monthly as of January 1966. Regular subscription rate: $\$ 72.00$ a year (6 Vols., 12 issues). Special rate: $\$ 36.00$ a year to individual members of supporting institutions.

Subscriptions, orders for back numbers, and changes of address should be sent to Pacific Journal of Mathematics, 103 Highland Boulevard, Berkeley, California, 94708.

PUBLISHED BY PACIFIC JOURNAL OF MATHEMATICS, A NON-PROFIT CORPORATION

Printed at Kokusai Bunken Insatsusha (International Academic Printing Co., Ltd.), 8-8, 3-chome, Takadanobaba, Shinjuku-ku, Tokyo 160, Japan.

Copyright (C) 1975 by Pacific Journal of Mathematics Manufactured and first issued in Japan 


\section{Pacific Journal of Mathematics}

\section{Vol. 63, No. 2 \\ April, 1976}

Joseph Anthony Ball and Arthur R. Lubin, On a class of contractive perturbations

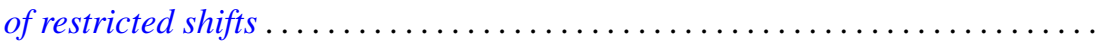

Joseph Becker and William C. Brown, On extending higher derivations generated

by cup products to the integral closure .......................

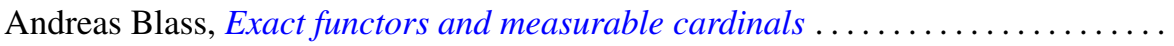

Joseph Eugene Collison, A variance property for arithmetic functions . . . . . . . . . .

Craig McCormack Cordes, Quadratic forms over nonformally real fields with a

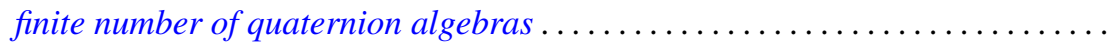

Freddy Delbaen, Weakly compact sets in $H^{1} \ldots \ldots \ldots \ldots \ldots \ldots \ldots \ldots \ldots$

G. D. Dikshit, Absolute Nörlund summability factors for Fourier series ..........

Edward Richard Fadell, Nielsen numbers as a homotopy type invariant. . ........

Josip Globevnik, Analytic extensions of vector-valued functions . . . . . . . . . . . .

Robert Gold, Genera in normal extensions . . . . . . . . . . . . . . . . . . . 389

Solomon Wolf Golomb, Formulas for the next prime

Robert L. Griess, Jr., The splitting of extensions of $S L(3,3)$ by the vector space

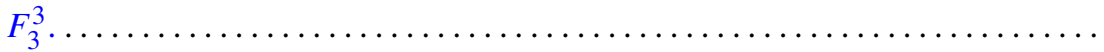

Thomas Alan Keagy, Matrix transformations and absolute summability .........

Kazuo Kishi, Analytic maps of the open unit disk onto a Gleason part.

Kwangil Koh, Jiang Luh and Mohan S. Putcha, On the associativity and commutativity of algebras over commutative rings . ..... . .

James C. Lillo, Asymptotic behavior of solutions of retarded differential difference equations.

John Alan MacBain, Local and global bifurcation from normal eigenvalues ..

Anna Maria Mantero, Sets of uniqueness and multiplicity for $L^{p}$

J. F. McClendon, Embedding metric families

L. Robbiano and Giuseppe Valla, Primary powers of a prime ideal .

Wolfgang Ruess, Generalized inductive limit topologies and barrelledness

properties.

Judith D. Sally, Bounds for numbers of generators of Cohen-Macaulay ideals

Helga Schirmer, Mappings of polyhedra with prescribed fixed points and fixed point indices.

Cho Wei Sit, Quotients of complete multipartite graphs

S. Sznajder and Zbigniew Zielezny, Solvability of convolution equations in $\mathscr{K}_{p}^{\prime}$,

$p>1$.

Mitchell Herbert Taibleson, The existence of natural field structures for finite

dimensional vector spaces over local fields

William Yslas Vélez, A characterization of completely regular fields

P. S. Venkatesan, On right unipotent semigroups ..............

Kenneth S. Williams, A rational octic reciprocity law ............

Robert Ross Wilson, Lattice orderings on the real field .......... 